\title{
Haemorrhagic Cystitis and Renal Dysfunction
}

\author{
Simone Cesaro
}

\subsection{Haemorrhagic Cystitis}

\subsubsection{Introduction}

Haemorrhagic cystitis (HC) is a frequent complication after HSCT. According to the time of occurrence after HSCT, HC is defined as earlyonset and late-onset. Early-onset HC occurs typically during or within $48 \mathrm{~h}$ after the end of conditioning regimen, and it is the result of a direct toxic effect of drug metabolites and radiotherapy on the bladder mucosa. Late-onset $\mathrm{HC}$ usually starts around the time of the period of neutrophil engraftment (weeks 2-4) or in second-third month after HSCT (Hirsch and Pergam 2016). The concurrent presence of prohaemorrhagic abnormalities of coagulation, severe thrombocytopenia and mucosal inflammation are predisposing factors for any type of $\mathrm{HC}$.

\subsubsection{Risk Factors}

The main risk factor for late-onset $\mathrm{HC}$ is infection by polyomavirus $\mathrm{BK}(\mathrm{BKPyV})$, whereas other viruses such as ADV, CMV, and HHV6 have been rarely implicated.

S. Cesaro $(\bowtie)$

Pediatric Hematology Oncology, Azienda

Ospedaliera Universitaria Integrata, Verona, Italy

e-mail: simone.cesaro@aovr.veneto.it
BKPyV is a common cause of asymptomatic or mild flu-like infection during early infancy and childhood, and more than $90 \%$ of adults are seropositive for BKPyV. The route of transmission is not clearly defined, but it is thought to be through oral saliva or respiratory tract secretions. After the primary infection, the virus persists latently in renal tubular epithelial and urothelial cell and can replicate as the host immune control loses the capacity to mount a virus-specific T-cell response. Mild to moderate asymptomatic BKPyV viruria is seen in $5-10 \%$ of healthy individuals, especially the oldest and the pregnant women, whereas high-load BKPyV viruria is detected in $50-60 \%$ of patients who underwent an allo-HSCT due to the delayed recovery of immune responses and the use of immunosuppressive drugs.

\subsubsection{Pathogenesis}

The current pathogenetic model of $\mathrm{HC}$ following HSCT is multifactorial and includes the combined effects of the extensive cytopathic damage of bladder mucosa layer due to the high-rate replicating virus, the chemical or actinic damage induced by conditioning regimen, and the donor-cell immune alloreactivity targeting bladder mucosa (Cesaro et al. 2018). In patients receiving allogeneic HSCT, both BKPyV viruria and viremia are specific and sensitive predictive factors for BKPyV-HC: a urine BKPyV load $>1 \times 10^{7}$ genomic copies $/ \mathrm{mL}$ had a 
sensitivity of $86 \%$ and specificity of $60 \%$, while a blood BKPyV load $>1 \times 10^{3}$ genomic copies $/ \mathrm{mL}$ had a sensitivity of $100 \%$ and a specificity of $86 \%$ for HC (Cesaro et al. 2015).

BKPyV infection develops in more than 50\% of allo-HSCT in the peri-engraftment weeks, but overt HC occurs in about $20 \%$ of patients because its development is influenced by the presence of several other risk factors: the type of graft (CB and PB vs. $\mathrm{BM}$ ); the type of donor (URD vs. MRD); the type of conditioning regimen (MAC vs. RIC); the use in the conditioning regimen of ATG, CY, or BU; the occurrence of acute GVHD grade 2-4; and, among the paediatric patients, a recipient age $>7$ years.

\subsubsection{Diagnosis}

The clinical diagnosis of BKPyV-HC is based on the presence of clinical symptoms/signs of cystitis, such as dysuria, increased urinary frequency, and lower abdominal pain, the presence of macrohaematuria, and the demonstration of BKPyV viruria, with viral loads of $>7 \log 10$ copies/mL.

The severity of haematuria is described by four categories: microscopic (grade 1), macroscopic (grade 2), macroscopic with clots (grade 3 ), and macroscopic with clots and renal failure secondary to urinary tract obstruction.

$\mathrm{BKPyV}$ viremia is often detected in patients with BKPyV-HC, and plasma viral load of $>3$ to $4 \log 10$ copies $/ \mathrm{mL}$ has been reported in more than two thirds of patients (Erard et al. 2005; Cesaro et al. 2015).

The reduction of both BKPyV viruria and $\mathrm{BKPyV}$ viremia has been correlated with clinical recovery from HC. Despite this, screening of asymptomatic HSCT patients at risk for $\mathrm{BKPyV}$ viruria and viremia remains an area of investigation and is presently not recommended since pre-emptive therapy is not established.

\subsubsection{Prophylaxis}

Effective preventive measures are available only for early-onset $\mathrm{HC}$, and they are mainly unspecific such as hyperhydration and the use of mesna in patients who receive high dose of $\mathrm{CY}$ as part of the conditioning regimen. The capacity of CY and its metabolite acrolein to damage the bladder mucosa determining an inflammatory reaction is well-known, and both hyperhydration and mesna reduce the exposure of bladder mucosa to acrolein and other toxic catabolites.

In line with this, the recent increase of unmanipulated haplo-HSCT with the administration of a PT-CY as prophylaxis of GVHD has resulted in a high incidence of HC. The use of bladder irrigation through a two- or three-way urinary catheter resulted not more effective in preventing $\mathrm{HC}$ compared to hyperhydration, and considering its invasiveness and discomfort for the patient, its use is not recommended.

In the pathogenetic model of late-onset $\mathrm{HC}$, BKPyV replication has a key role in exacerbating the damage of bladder mucosa through its cytopathic effect and in inducing the donor immune alloreactivity to target the bladder mucosa. In order to reduce BKPyV replication, ciprofloxacin has been used prophylactically by several authors because of the in vitro capacity of fluoroquinolones to inhibit BKPyV replication. Overall, the efficacy of ciprofloxacin was weak and limited to a reduction of $\mathrm{BKPyV}$ replication without significantly affecting the incidence of HC. Considering the risk of inducing bacterial resistance and the risk of tendinitis and joint damage in children, the use of fluoroquinolones is not recommended for this purpose.

\subsubsection{Treatment}

$\mathrm{BKPyV}$ replication can be controlled more effectively by cidofovir, a nucleotide analogue inhibiting several DNA viruses such as CMV, ADV, HHV6, HSV, HVZ, and smallpox. The important pharmacokinetic property of cidofovir is the long half-life of its active metabolites ranging from 15 to $65 \mathrm{~h}$ that allows the administration on a weekly basis. Given the significant risk of tubular nephrotoxicity, cidofovir has been used only for therapeutic purposes (Cesaro et al. 2009). The nephrotoxicity can be limited by saline hydration and by the use of probenecid that inhibits the capture and transport of cidofovir into the tubular 
epithelial cells of the kidney. Despite that several authors have assessed cidofovir as treatment of $\mathrm{BKPyV}-\mathrm{HC}$, there is no agreement on the optimal dose, modality of administration, and frequency of administration. Most authors use intravenous cidofovir at the dose of $3-5 \mathrm{mg} / \mathrm{kg} /$ weekly or fortnightly together with probenecid to prevent nephrotoxicity obtaining a complete clinical response in $74 \%$ of patients and at least $1 \log$ decline in urine and blood viral loads in $38 \%$ and $84 \%$ of patients. As expected, the main adverse effect was renal toxicity with a mild to moderate increase in serum creatinine observed in $18 \%$ of the patients. The second more frequent scheme of treatment was a dose of cidofovir of $0.5-1.5 \mathrm{mg} /$ $\mathrm{kg}$ without probenecid, administered 1-3 times a week (Ganguly et al. 2010). A complete clinical response was observed in $83 \%$ of patients, with a significant reduction of viral load in the urine and in the blood in $62 \%$ and $67 \%$ of patients, respectively. Also, with this schedule, mild to moderate renal toxicity was reported in $20 \%$ of patients. An alternative route that can reduce the risk of nephrotoxicity is the administration of cidofovir intravesically. Although the experience is limited to a small number of patients, the dose of $5 \mathrm{mg} / \mathrm{kg} /$ week, left in situ for 1-2 $\mathrm{h}$ after clamping the vesical catheter, showed an overall clinical response in $43 \%$ of patients and a virological response in about 50\% (Bridges et al. 2006).

Preliminary encouraging results have also been obtained with leflunomide, an antimetabolite drug with immunomodulatory and antiviral activity, whereas a successful treatment has been reported anecdotal and in older series with vidarabine, oral levofloxacin, FXIII concentrate, intravesical sodium hyaluronate, and oestrogens (Cesaro et al. 2018).

The recovery from HC, whatever the cause, can benefit from treatment aiming to repair and regenerate the urothelial mucosa, such as hyperbaric oxygen therapy and the topical application of fibrin glue. Although experienced in a limited number of patients, the use of hyperbaric oxygen therapy was associated with a complete clinical response rate of $86 \%$ and a reduced urine $\mathrm{BKPyV}$ load in $65 \%$ of patients. The main drawback of hyperbaric oxygen is the limited availability, the requirements for dedicated hyperbaric room facilities, the risk of ear barotrauma or pressure intolerance, and claustrophobia episodes during the procedure (Zama et al. 2013; Cesaro et al. 2018). Cystoscopic application of fibrin glue to the damaged bladder mucosa to achieve haemostasis has been reported in single-centre retrospective series with a complete response rate was $83 \%$, with most of cases resolved with just one or two applications (Tirindelli et al. 2014).

Given the important role of immune response in the pathogenesis of late-onset $\mathrm{HC}$ and in absence of effective antiviral drugs, innovative therapies have been trialled, such as the use of mesenchymal stromal cells (MSC) and adoptive immunotherapy. MSC have the potential to stimulate the tissue repairing process and exercise an immune modulatory and anti-inflammatory effect. The use of third party MSC infusion into seven patients with BKPyV-HC obtained the resolution of haematuria in five patients (Ringden et al. 2007). This approach needs to be validated further to assess the feasibility and also the safety of MSC.

Adoptive transfer of donor-derived virusspecific T cells (VSTs) has shown efficacy for the treatment of several viral infections although their use on a larger scale is limited by the costs, the complexity of manufacturing, the time needed to obtain the final cell product that is not suitable for the urgent treatment, and the prompt availability of a seropositive donor. The use of banked VSTs with multiple specificity obtained by a third party healthy seropositive donor cryopreserved and used as the patient developed a viral infection refractory to antiviral treatment represents a promising development. In a phase II trial, the use of VSTs directed against five viruses, CMV, EBV, ADV, HHV-6, and BKPyV, obtained an overall cumulative response rate of $92 \%$. The virological response for BKPyV was $100 \%$, and 13 of 14 patients treated for $\mathrm{HC}$ had a resolution of haematuria by 6 weeks. The infusions of VSTs resulted safe, and only 2 of 45 infusions were followed by a mild GVHD reaction (grade 1). Importantly, the functionality of VSTs persisted for up 12 weeks (Tzannou et al. 2017). These results are encouraging and support further studies. 


\subsection{Renal Dysfunction}

Acute kidney injury (AKI) occurs in 27-66\% of patients who underwent allo-HSCT mainly within the first 100 days. The incidence of AKI in less than $20 \%$ in auto-HSCT due to several reasons such as the rapid engraftment and reduced cytopenia especially with PBSC grafts, the lower incidence of infectious complications, and the absence of GVHD that can determine a renal damage directly by inducing inflammatory cytokines, CMV reactivation, diarrhoea with severe dehydration, and indirectly, through the nephrotoxicity of the drugs used to prevent or treat GVHD such as CNI and high-dose PRD (Lopes et al. 2016; Raina et al. 2017).

The diseases associated with AKI act at different renal levels: prerenal (sepsis, engraftment syndrome, SOS/VOD), renal-glomerular (transplant-associated microangiopathy), renaltubular (acute tubular necrosis due to dehydration, sepsis, shock, engraftment syndrome, intratubular obstruction due to drugs, or tumour lysis syndrome), renal-interstitial (acute GVHD, viral infection by BKPvyV or ADV), and postrenal (obstruction by BKPyV or adenovirus cystitis, retroperitoneal fibrosis, lymphadenopathy).
Moreover, there are general favouring factors for AKI such as the presence pre-HSCT of diabetes, hypertension, and renal impairment as well as the use of nephrotoxic drugs in the conditioning regimen (ifosphamide, CY, carboplatin, cisplatin), for treatment of GVHD (MTX, $\mathrm{CNI}$ ), for the treatment of infections (AmB, aminoglicosydes, vancomycin), and for other severe organ damages that require ICU admission and mechanical ventilation (Hingorani 2016).

Clinically, the severity of AKI is defined by the measure of serum creatinine ( $\mathrm{SCr}$ ) and urine output (UO) that permit the identification of three classes: risk of AKI (increase of SCr 1.5$2 x$ and $\mathrm{UO}<0.5 \mathrm{~mL} / \mathrm{kg} / \mathrm{h}$ for $>6 \mathrm{~h}$ ), kidney injury (increase of SCr $2-3 \times$, and $\mathrm{UO}<0.5 \mathrm{~mL} /$ $\mathrm{kg} / \mathrm{h}$ for $>12 \mathrm{~h}$ ), and kidney failure (increase of $\mathrm{SCr}>3 \times$ and $\mathrm{UO}<0.3 \mathrm{~mL} / \mathrm{kg} / \mathrm{h}$ for $>24 \mathrm{~h}$ or anuria $>12 \mathrm{~h}$, or initiation of replacement therapy) (Lopes et al. 2016). AKI represents a risk factor for the development on the medium-longterm period of chronic kidney disease, especially if the acute damage is not completely resolved and proteinuria and hypertension persist and for increased of non-relapse and overall mortality (Shingai et al. 2015).

\begin{tabular}{|c|c|c|c|}
\hline & Early-onset HC & Late-onset $\mathrm{HC}$ & Comments \\
\hline Incidence & $<3 \%$ & $7-25 \%$ & $\begin{array}{l}\text { Early-onset } \mathrm{HC} \text { is nowadays } \\
\text { rare }\end{array}$ \\
\hline Pathogenesis & $\begin{array}{l}\text { Chemical or actinic damage of } \\
\text { bladder mucosa }\end{array}$ & $\begin{array}{l}\text { - BKPyV infection } \\
\text { - Adenovirus infection } \\
\text { - Donor alloreactivity }\end{array}$ & \\
\hline Diagnosis & $\begin{array}{l}\text { Macrohaematuria with } \\
\text { dysuria, increased urinary } \\
\text { frequency, low abdominal } \\
\text { pain }\end{array}$ & $\begin{array}{l}\text { Macrohaematuria with } \\
\text { dysuria, increased urinary } \\
\text { frequency, low abdominal } \\
\text { pain, high load of BKPyV } \\
\text { urine and/or plasma }\end{array}$ & $\begin{array}{l}\text { Signs of bladder inflammation } \\
\text { at ultrasound examination }\end{array}$ \\
\hline Prevention & $\begin{array}{l}\text { Hyperhydration, mesna (if } \\
\text { CY), forced diuresis }\end{array}$ & $\begin{array}{l}\text { Hyperhydration, forced } \\
\text { diuresis }\end{array}$ & $\begin{array}{l}\text { Fluoroquinolones not } \\
\text { recommended }\end{array}$ \\
\hline Therapy & $\begin{array}{l}\text { - Hyperhydration } \\
\text { - Forced diuresis } \\
\text { - Hyperbaric O2 } \\
\text { - Application of fibrin glue by } \\
\text { cystoscopy }\end{array}$ & $\begin{array}{l}\text { - Hyperhydration, forced } \\
\text { diuresis } \\
\text { - IV (or intravesical) } \\
\text { cidofovir } \\
\text { - Hyperbaric O2 therapy } \\
\text { - Application of fibrin glue } \\
\text { by cystoscopy }\end{array}$ & $\begin{array}{l}\text { - Cidofovir: No agreement on } \\
\text { dose and route of admin } \\
\text { Limited evidence } \\
\text { - O2 and fibrin glue limited } \\
\text { experience }\end{array}$ \\
\hline Experimental & I & $\begin{array}{l}\text { - Mesenchymal cells } \\
\text { - Virus-specific T cells }\end{array}$ & \\
\hline
\end{tabular}




\section{References}

Bridges B, Donegan S, Badros A. Cidofovir bladder instillation for the treatment of BK hemorrhagic cystitis after allogeneic stem cell transplantation. Am J Hematol. 2006;81:535-7.

Cesaro S, Hirsch HH, Faraci M, et al. Cidofovir for BK virus-associated hemorrhagic cystitis: a retrospective study. Clin Infect Dis. 2009;49:233-40.

Cesaro S, Tridello G, Pillon M, et al. A prospective study on the predictive value of plasma BK virus-DNA load for hemorrhagic cystitis in pediatric patients after stem cell transplantation. J Pediatric Infect Dis Soc. 2015;4:134-42.

Cesaro S, Dalianis T, Hanssen Rinaldo C, et al. ECIL guidelines for the prevention, diagnosis and treatment of BK polyomavirus-associated haemorrhagic cystitis in haematopoietic stem cell transplant recipients. J Antimicrob Chemother. 2018;73:12-21.

Erard V, Kim HW, Corey L, et al. BK DNA viral load in plasma: evidence for an association with hemorrhagic cystitis in allogeneic hematopoietic cell transplant recipients. Blood. 2005;106:1130-2.

Ganguly N, Clough LA, Dubois LK, et al. Low-dose cidofovir in the treatment of symptomatic BK virus infection in patients undergoing allogeneic hematopoietic stem cell transplantation: a retrospective analysis of an algorithmic approach. Transpl Infect Dis. 2010;12:406-11.

Hingorani S. Renal complications after hematopoietic stem cell transplantation. N Engl J Med. 2016; 374:2256-67.

Hirsch HH, Pergam SA. Human adenovirus, polyomavirus, and parvovirus infections in patients undergoing hematopoietic stem-cell transplantation. In: Forman SJ, Nagrin RS, Antin H, Appelbaum FR, editors. Thomas' hematopoietic cell transplantation. 5th ed. Hoboken: Wiley; 2016. p. 1090-104. http://www. wiley.comgoappelbaumTransplantation.

Lopes JA, Jorge S, Neves M. Acute kidney injury in HCT: an update. Bone Mmarrow Ttransplant. 2016;51:755-62.

Raina R, Herrera N, Krishnappa V, et al. Hematopoietic stem cell transplantation and kidney injury in children: a comprehensive review. Pediatr Transplant. 2017;21:e12935.

Ringden O, Uzunel M, Sundberg B, et al. Tissue repair using allogeneic mesenchymal stem cells for hemorrhagic cystitis, pneumomediastinum and perforated colon. Leukemia. 2007;21:2271-6.

Shingai N, Morito T, Najima Y, et al. Early-onset acute kidney injury a poor prognostic sign for allogeneic SCT recipients. Bone Marrow Transplant. 2015;50:1557-662.

Tirindelli MC, Flammia GP, Bove P, et al. Fibrin glue therapy for severe hemorrhagic cystitis after allogeneic hematopoietic stem cell transplantation. Biol Blood Marrow Transplant. 2014;20:1612-7.

Tzannou I, Papadopoulou A, Naik S, et al. Off-the-shelf virus specific $\mathrm{T}$ cells to treat $\mathrm{BK}$ virus, human herpes virus 6, cytomegalovirus, Epstein-Barr virus, and adenovirus infections after allogeneic hematopoietic stem cell transplantation. J Clin Oncol. 2017;35:3547-57.

Zama D, Masetti R, Vendemini F, et al. Clinical effectiveness of early treatment with hyperbaric oxygen therapy for severe late-onset hemorrhagic cystitis after hematopoietic stem cell transplantation in pediatric patients. Pediatr Transplant. 2013;17:86-91.

Open Access This chapter is licensed under the terms of the Creative Commons Attribution 4.0 International License (http://creativecommons.org/licenses/by/4.0/), which permits use, sharing, adaptation, distribution and reproduction in any medium or format, as long as you give appropriate credit to the original author(s) and the source, provide a link to the Creative Commons license and indicate if changes were made.

The images or other third party material in this chapter are included in the chapter's Creative Commons license, unless indicated otherwise in a credit line to the material. If material is not included in the chapter's Creative Commons license and your intended use is not permitted by statutory regulation or exceeds the permitted use, you will need to obtain permission directly from the copyright holder. 